\title{
An Urban Center Experience Exploring Barriers to Adherence to Endoscopic Surveillance for Non- Dysplastic Barrett's Esophagus
}

Mahmoud Isseh $^{1}$, Laurel Mueller ${ }^{2}$, Hussna Abunafeesa ${ }^{2}$, Zaid Imam ${ }^{3}$, Dania Shakaroun ${ }^{2}$, Mouhanna Abu Ghanimeh ${ }^{4}$, Nazih Isseh ${ }^{5}$, Joseph Miller ${ }^{6}$, Syed-Mohammed Jafri ${ }^{4}$, Adrienne Lenhart ${ }^{7}$

1. Internal Medicine, University of Michigan, Ann Arbor, USA 2. Internal Medicine, Henry Ford Health System, Detroit, USA 3. Gastroenterology and Hepatology, William Beaumont Hospital, Royal Oak, USA 4. Gastroenterology and Hepatology, Henry Ford Health System, Detroit, USA 5. Internal Medicine, The University of Tennessee Health Science Center, Memphis, USA 6. Emergency Medicine, Henry Ford Health System, Detroit, USA 7. Gastroenterology, University of California Los Angeles, Los Angeles, USA

Corresponding author: Mahmoud Isseh, mahmoudisseh89@gmail.com

\section{Abstract \\ Background}

Data regarding barriers to Barrett's esophagus (BE) surveillance is limited. Studying an urban center population, we aimed to characterize non-dysplastic BE surveillance rates and identify health, racial, and socioeconomic disparities affecting surveillance.

\section{Methods}

Patients with biopsy-confirmed BE were retrospectively identified between January 2002 and December 2012. Non-dysplastic BE patients were analyzed for adherence to established surveillance guidelines. Demographic, racial, comorbidities, and socioeconomic variables were extracted. Annual gross income (AGI) was utilized as a marker of socioeconomic status (SES). Univariate and multivariate analyses compared adherent vs. non-adherent patients to surveillance guidelines.

\section{Results}

A total of 217 patients with non-dysplastic BE were analyzed. The majority were male (67.3\%) and Caucasian (75.6\%), with only $47.5 \%$ adherent with the first surveillance endoscopy. Patients with a high average AGI were more likely to be adherent with the initial surveillance endoscopy than those with low AGI ( $\mathrm{p}=0.032)$. Initial compliance with first surveillance was associated with better surveillance at regular intervals $(\mathrm{p}=0.001)$. No significant differences in age, primary language, insurance type, marital status, or Charlson Comorbidity Index (CCI) between adherent and non-adherent patients were found.

Review began 01/16/2021 Review ended 01/27/2021 Published 01/31/2021

๑) Copyright 2021

Isseh et al. This is an open access article distributed under the terms of the Creative Commons Attribution License CC-BY 4.0., which permits unrestricted use, distribution, and reproduction in any medium, provided the original author and source are credited.

\section{Conclusions}

Although overall adherence to guidelines was suboptimal, this study identifies important socioeconomic disparities in the endoscopic surveillance for non-dysplastic BE. Identifying and understanding the barriers to care among these lower socioeconomic groups may ultimately lead to improved screening compliance and early BE detection.

Categories: Gastroenterology

Keywords: barrett's esophagus, surveillance, barrett's dysplasia

\section{Introduction}

Barrett's esophagus (BE) is a significant precursor to the development of esophageal adenocarcinoma (EAC), which occurs in approximately $0.2 \%-7 \%$ of patients with BE each year, depending on the degree of dysplasia $[1,2]$. Given the increased risk of malignancy, guidelines recommend endoscopic surveillance of BE to help increase the likelihood of early cancer detection and improve overall survival [2]. However, several retrospective and survey studies have shown poor adherence to established guidelines, with only $26 \%-77 \%$ of endoscopists demonstrating compliance [3-10]. BE has also been shown to have a higher prevalence among older, Caucasian, men, with lower rates among Hispanic, Asian, and African American patients [9,1113].

Prior studies have suggested that lower socioeconomic status (SES) is associated with lower rates of colorectal, breast, and cervical cancer screening [14,15]. Additionally, higher EAC stage at the time of diagnosis has been reported in lower SES populations [16]. Less awareness, lack of knowledge and support, negative beliefs about malignancy screening, and ideas about cancer fatality are some of the psychosocial aspects studied impacting attitudes of individuals of lower SES towards cancer screening [15]. Adherence 
with surveillance guidelines for BE is poor in clinical practice, and disparities in the demographic distribution of BE have been identified. However, data is limited pertaining to existing barriers to surveillance. A recent study by Dalal et al. assessed adherence to endoscopic surveillance guidelines in nondysplastic BE patients in a Veterans Affairs (VA)-based population, and found that adherent cases were more likely to be older in age with a trend towards having long segment BE on initial endoscopy [10]. Using an urban center population, we aimed to characterize non-dysplastic BE surveillance rates and identify health, racial, and socioeconomic disparities potentially affecting surveillance.

\section{Materials And Methods}

We performed a retrospective chart review of patients with biopsy-confirmed BE, identified from January 2002 through December 2011 using the International Classification of Diseases (ICD) 9 and ICD 10 codes. This study was conducted at a high-volume, urban, tertiary care hospital, only after approval from the Institutional Review Board. Patients included in this study were 18 years or older and were identified to have either non-dysplastic, low-grade dysplastic, or high-grade dysplastic BE on index upper endoscopy. Patients over the age of 75 years, patients with presumed BE but without biopsy confirmation, patients with indefinite biopsy results, or patients with EAC on initial endoscopy were excluded. Additionally, patients diagnosed with $\mathrm{BE}$ at an outside institution were excluded given the inability to confirm endoscopy and histopathological reports in the electronic medical record. Non-dysplastic BE patients were then further analyzed to determine adherence with established guidelines.

The primary outcome of our study was to determine the rate of adherence to surveillance guidelines in patients with non-dysplastic BE. A patient's index endoscopy was defined as the first documented esophagogastroduodenoscopy (EGD) in our hospital system with esophageal biopsies that confirmed nondysplastic BE. Adherence to surveillance guidelines was defined as a follow-up endoscopy after three years if the initial endoscopy was performed between 2002-2010, or after 3-5 years for index endoscopies performed during or after 2011. These different BE surveillance intervals were chosen based upon a change in the American College of Gastroenterology’s (ACG) and American Gastroenterological Association's (AGA) established guidelines during this time frame. Both the 2002 and 2008 ACG practice guidelines for BE recommend a follow-up endoscopy after three years for patients with non-dysplastic BE [17,18]. However, the AGA medical position statement on the management of BE in 2011 recommends a follow-up endoscopy after three to five years for patients with non-dysplastic BE [19]. The follow-up interval of 2002 through 2011 was selected in order to analyze a decade of follow-up and surveillance for BE. A window period of six months on either side of the three year or three to five year follow-up period was allowed to count towards adherence. In cases in which a confirmatory endoscopy was performed within 2.5 years of the index EGD, this endoscopy did not count towards surveillance.

Secondary outcomes of our study were to determine if initially, adherent patients were more likely to undergo continued surveillance at regular or irregular intervals, as well as to determine any racial, social, or financial variables that could predict adherence to BE surveillance guidelines. The duration of additional surveillance was characterized from the first surveillance endoscopy through December of 2017. Regular surveillance was defined as continued adherence to guidelines based on the results of each additional esophageal biopsy [17-19]. Irregular surveillance was defined as having either no additional surveillance endoscopies or endoscopies at periods other than those recommended by guidelines. Patients who died during the follow-up period or had their first surveillance endoscopy performed during or after 2012 were excluded from this part of the analysis.

Patient variables, including age, gender, race, body mass index (BMI), primary language, marital status, insurance type, annual gross income (AGI), and the Charlson Comorbidity Index (CCI) were collected and analyzed. AGI was determined based on the average household income of patients residing in specific zip codes and ranged from less than 40K, 40-60K, 60-80K, 80-100K, to over 100K United States Dollars (USD). An online website providing this data based on United States census bureau data was utilized to extract average household incomes used to define AGI [20]. These variables were then assessed to determine if any disparities existed between those non-dysplastic BE patients who were adherent vs. non-adherent to surveillance guidelines. Only adherence to the initial surveillance endoscopy was analyzed in this portion of the study. Health Alliance Plan (HAP) is a non-profit organization in Detroit, Michigan providing multiple health maintenance organization (HMO) and preferred provider organization (PPO) coverage plans to patients [21].

\section{Statistical analysis}

Comparison testing was performed using the Fisher exact test and Chi square test for categorical variables, two-sample t-tests for normally distributed numeric variables, and Wilcoxon rank sum tests for nonnormally distributed numeric variables. A multivariate logarithmic regression model included all variables predicting adherence with two-sided $\mathrm{p}$-values $<0.25$. Statistical significance was defined as a two-sided $\mathrm{p}$ value of $<0.05$ for all tests. Analyses were performed using Statistical Package for the Social Sciences; version 9.4 (SPSS Inc., Chicago, IL).

\section{Results}




\section{Cureus}

\section{Demographics}

We reviewed patients who carried a diagnosis of BE. Patients over the age of 75 years, patients without esophageal biopsies confirming BE, patients diagnosed with BE at an outside institution, patients with indefinite esophageal biopsy results, and patients with EAC on initial endoscopy were excluded from the analysis. A total of 224 patients met the inclusion criteria. Of these subjects, five patients had BE with lowgrade dysplasia, two patients had BE with high-grade dysplasia and 217 patients had non-dysplastic BE. Since the management of individuals with low-grade and high-grade dysplasia is different in terms of surveillance duration and endoscopic therapy, these were excluded from the analyzed cohort. No subanalysis was performed given the small number of patients with low-grade and high-grade dysplasia rendering analysis of low utility. Hence, 217 patients with non-dysplastic BE were in the final analysis. The mean age of individuals at the time of index endoscopy was $63.98 \pm 10.13$ years, and the majority were male (67.28\%) and Caucasian (75.58\%). In addition, the majority of patients in this study were married (63.59\%), spoke English as their primary language (94.93\%), had HAP for insurance coverage (40.09\%), and had shortsegment BE on esophageal biopsy (70.97\%). Additional characteristics of the study population are described in Table 1.

\section{Variable}

Mean Age $\pm S D$, years

Mean Charlson Comorbidity Index \pm SD

Gender

Male Sex

Race

Caucasian

African American

Asian

Unknown

Primary Language

English

Other

Unknown

Type of Health Insurance

Health Alliance Plan

Private, Non-Health Alliance Plan

Medicare

Medicaid

None

Marital Status

Married

Single

Comorbidities

Hypertension

Diabetes Mellitus

Obesity

Liver Disease

Chronic Obstructive Pulmonary Disease
$77(35.48 \%)$

$151(69.59 \%)$

$57(26.27 \%)$

$71(32.72 \%)$

$15(0.31 \%)$

Patient No. (\%)

$63.98 \pm 10.13$

$4.38 \pm 2.48$

$146(67.28 \%)$

$164(75.58 \%)$

$37(17.05 \%)$

$(2.30 \%)$

$11(5.07 \%)$

$206(94.93 \%)$

$4(1.84 \%)$

$7(2.83 \%)$

$87(40.09 \%)$

$49(22.58 \%)$

$69(31.80 \%)$

$5(2.30 \%)$

$4(1.84 \%)$

$31(14.29 \%)$ 


\section{Cureus}

Congestive Heart Failure

$22(10.14 \%)$

Coronary Artery Disease

$23(10.60 \%)$

Chronic Kidney Disease

$35(16.13 \%)$

Malignancy

$64(29.49 \%)$

Annual Gross Income

$<40 \mathrm{~K}$

$56(25.81 \%)$

40-60K

$75(34.56 \%)$

$60-80 \mathrm{~K}$

$41(18.89 \%)$

80-100K

$13(5.99 \%)$

$>100 \mathrm{~K}$

$24(11.06 \%)$

TABLE 1: Patient demographics

$\mathrm{K}$, thousands; SD, standard deviation.

\section{Adherence to surveillance guidelines}

Of the 217 patients with non-dysplastic BE, only 103 (47.47\%) were adherent with the first recommended surveillance endoscopy, whereas 114 (52.53\%) were non-adherent with surveillance guidelines. Of the 103 patients who were adherent with the initial surveillance endoscopy, 35 patients had continued surveillance endoscopies at regular intervals, 28 patients proceeded with irregular surveillance, and 17 patients had no additional surveillance endoscopies. Four patients were excluded from this portion of the analysis secondary to death during the follow-up period confirmed through the medical record, and 19 patients were excluded secondary to undergoing their first surveillance endoscopy during or after 2012. Of the 114 patients with non-dysplastic BE who were non-adherent with the first surveillance endoscopy, nine patients had continued regular surveillance endoscopies, 28 patients proceeded with irregular surveillance, and 56 patients had no additional surveillance endoscopies. Ten patients were excluded from this portion of the analysis secondary to death during the follow-up period, and 11 patients were excluded secondary to undergoing their first surveillance endoscopy during or after 2012. Patients who were adherent to their first surveillance endoscopy were more likely to undergo additional surveillance endoscopies at regular intervals than were patients who were non-adherent to their first surveillance endoscopy ( $p=0.001)$.

\section{Variables that may predict adherence to surveillance guidelines}

In univariate analysis, patient age, gender, marital status, primary language, annual office visit with a primary care provider, obesity, tobacco use, and alcohol use had no significant impact on predicting adherence to the first surveillance endoscopy in patients with non-dysplastic BE. In addition, the CCI and the individual patient comorbidities of hypertension, diabetes mellitus, chronic obstructive pulmonary disease, congestive heart failure, coronary artery disease, malignancy, and chronic kidney disease (CKD) also did not help to predict adherence to surveillance guidelines. While not statistically significant, we did observe a trend towards better adherence among Caucasian patients compared to non-Caucasian patients ( $51.83 \%$ of Caucasian patients were adherent compared to $35.71 \%$ of non-Caucasian patients; $\mathrm{p}=0.062$ ) (Table 2). 


\section{Cureus}

\begin{tabular}{|c|c|c|c|}
\hline Variable & Non-Adherent to Surveillance & Adherent to Surveillance & P-value \\
\hline & $\mathrm{N}=114$ & $\mathrm{~N}=103$ & \\
\hline Mean Age, years $\pm S D$ & $64.0 \pm 10.9$ & $63.9 \pm 9.4$ & 0.961 \\
\hline Mean CClıSD & $4.7 \pm 2.7$ & $4.2 \pm 2.2$ & 0.208 \\
\hline Male Sex & $76(66.7 \%)$ & $70(68.0 \%)$ & 0.839 \\
\hline Race & & & 0.062 \\
\hline Caucasian & $79(74.5 \%)$ & $85(85.0 \%)$ & \\
\hline Other & $27(25.5 \%)$ & $15(15.0 \%)$ & \\
\hline English primary language & $110(99.1 \%)$ & $96(97.0 \%)$ & 0.345 \\
\hline Married & $69(61.1 \%)$ & $69(67.6 \%)$ & 0.315 \\
\hline Annual PCP visit & $82(72.6 \%)$ & $78(75.7 \%)$ & 0.596 \\
\hline Tobacco Use & $56(49.1 \%)$ & $61(59.8 \%)$ & 0.116 \\
\hline Alcohol Use & $57(50.0 \%)$ & $52(51.0 \%)$ & 0.886 \\
\hline Hypertension & $81(71.1 \%)$ & $70(68.0 \%)$ & 0.621 \\
\hline Diabetes Mellitus & $31(27.2 \%)$ & $26(25.2 \%)$ & 0.744 \\
\hline Obesity & $36(31.6 \%)$ & $35(34.7 \%)$ & 0.632 \\
\hline COPD & $16(14.0 \%)$ & $15(14.6 \%)$ & 0.912 \\
\hline Congestive Heart Failure & $13(11.4 \%)$ & $9(8.7 \%)$ & 0.516 \\
\hline Coronary Artery Disease & $10(8.8 \%)$ & $13(12.6 \%)$ & 0.358 \\
\hline Liver Disease & $10(8.8 \%)$ & $5(4.9 \%)$ & 0.256 \\
\hline Malignancy (solid tumor) & $28(24.6 \%)$ & $31(30.1 \%)$ & 0.360 \\
\hline Chronic Kidney Disease & $23(20.2 \%)$ & $12(11.7 \%)$ & 0.088 \\
\hline Health Insurance Type & & & 0.369 \\
\hline Health alliance plan & 41 (36.3\%) & $46(45.5 \%)$ & \\
\hline Private, Non-HAP & $31(27.4 \%)$ & $18(17.8 \%)$ & \\
\hline Medicare & $35(31.0 \%)$ & 34 (33.7\%) & \\
\hline Medicaid & $3(2.7 \%)$ & $2(2.0 \%)$ & \\
\hline Non-insured & $3(2.7 \%)$ & $1(1.0 \%)$ & \\
\hline Annual Gross Income & & & $0.032^{\star}$ \\
\hline$<40 \mathrm{~K}$ & $36(32.1 \%)$ & $20(20.6 \%)$ & \\
\hline $40-60 \mathrm{~K}$ & 40 (35.7\%) & $35(36.1 \%)$ & \\
\hline $60-80 \mathrm{~K}$ & $21(18.8 \%)$ & $20(20.6 \%)$ & \\
\hline $80-100 \mathrm{~K}$ & $5(4.5 \%)$ & $8(8.2 \%)$ & \\
\hline$>100 \mathrm{~K}$ & $10(8.9 \%)$ & $14(14.4 \%)$ & \\
\hline
\end{tabular}

TABLE 2: Univariate analyses comparing variables between adherent and non-adherent groups to surveillance guidelines

N, Number; HAP, Health Alliance Plan; K, Thousands; COPD, chronic obstructive pulmonary disease; PCP, primary care physician; CCI, Charlson Comorbidity Index; SD, standard deviation. 
The type of health insurance that patients' possessed did not have any significant impact on predicting adherence to surveillance guidelines in patients with non-dysplastic BE. However, patients with a higher average AGI were more likely to be adherent with the initial surveillance endoscopy compared to patients with a lower average AGI (35.71\% of patients with an AGI <40K were adherent, $46.67 \%$ of patients with an AGI of $40-60 \mathrm{~K}$ were adherent, $48.78 \%$ of patients with an AGI of $60-80 \mathrm{~K}$ were adherent, $61.54 \%$ of patients with an AGI of $80-100 \mathrm{~K}$ were adherent, and $58.33 \%$ of patients with an AGI >100K were adherent; $p=0.032$ ) (Table 2).

Caucasian race, tobacco use, moderate to severe CKD, AGI, and CCI score were the variables included in multivariate analysis. A non-significant trend toward better adherence was noted among Caucasian patients $(\mathrm{p}=0.097)$ and patients with higher AGI $(\mathrm{p}=0.086)$. These results are summarized in Table 3.

\begin{tabular}{|c|c|c|c|c|}
\hline Variable & P-value & Odds Ratio & & Oonfidence Interval \\
\hline Caucasian Race & 0.097 & 1.911 & 0.890 & 4.105 \\
\hline Tobacco Use & 0.288 & 1.377 & 0.763 & 2.486 \\
\hline Moderate to Severe CKD & 0.620 & 0.796 & 0.324 & 1.958 \\
\hline Annual Gross Income & 0.086 & 1.229 & 0.972 & 1.555 \\
\hline CCl Score & 0.388 & 0.945 & 0.831 & 1.074 \\
\hline
\end{tabular}

TABLE 3: Multivariable logistic regression results using patient characteristics to predict adherence

Only variables with p-values less than 0.25 from Table 2 were included in this analysis.

CCI: Charlson Comorbidity Index; CKD: chronic kidney disease.

Of the 35 patients who continued surveillance endoscopies at regular intervals, $65.7 \%$ were male, $80 \%$ were Caucasian, their mean age was $60.5 \pm 10.2$ years, $68.6 \%$ had short segment $B E$, the mean number of additional endoscopies performed were $2 \pm 0.13$, none of these 35 patients developed EAC.

\section{Discussion}

In this busy tertiary care hospital-based study of 217 patients with biopsy-proven non-dysplastic BE, we found that less than half of the cases (47.47\%) were adherent with guidelines. Our results are similar to Dalal et al.'s rate of 30\% guideline adherence for non-dysplastic BE [10]. It is important to note that our guideline adherence rates which are based on a retrospective review of cases with biopsy-proven non-dysplastic BE are significantly lower than the rates claimed by gastroenterologists in surveys conducted both in the US and Europe ( $86 \%$ and $76 \%$, respectively) $[22,23]$.

Our study showed that $43.68 \%$ of the patients who got the initial surveillance endoscopy either continued with irregular surveillance or failed to get any additional surveillance endoscopies (28 cases and 17 cases, respectively-out of the 103 cases with initial endoscopy). It is unclear whether physicians have a role in this trend in our healthcare system, as many gastroenterologists who have poor surveillance rates claim lack of efficacy of surveillance [8]. This is especially true with non-dysplastic BE which has a very low annual incidence of EAC (0.33\%), and even lower with short-segment BE (0.19\%) [2] which represents $70.97 \%$ of all our biopsy-proven BE.

Although many variables including type of insurance, annual office visit with a primary care provider, and patients' comorbidities failed to predict adherence to surveillance guidelines, it is noted that Caucasian patients were more likely to adhere to a first surveillance endoscopy than non-Caucasian patients $51.83 \%$ and $35.71 \%$ retrospectively; $\mathrm{p}=0.062$ ). It is important to note that over the last few decades, several studies have reported the rarity of $\mathrm{BE}$ and EAC diagnosis in the African American population which encompasses most of our non-Caucasian patients [12]. This was indeed true in our study where $75.58 \%$ of the patients with non-dysplastic BE on biopsy were Caucasians and only 17.05\% were African Americans (Table 1). Despite a lower rate of BE and dysplastic BE in African Americans compared to non-Hispanic whites [12], the variation in rates of adherence to guidelines can be multifactorial. Mistrust of the medical community, limited access to care, lack of social support, and poor health literacy can be contributors to the lower rates of surveillance endoscopies in the non-Caucasians and mainly the African American population.

Our study corroborated the negative impact of lower SES to adherence to screening programs, similar to other studies evaluating colorectal and breast cancer [24,25]. Establishing national screening programs do 
not ameliorate the effects of these disparities on earlier diagnosis of malignancy [26]. Multifactorial mechanisms including psychosocial factors impacting access and adherence of individuals from disadvantaged populations translate into worse patient outcomes [15,16]. Identifying these disparities and more in-depth analysis of the etiological factors behind them is the first step in improving the adherence of disadvantaged populations to screening programs.

The study's strengths include its ability to evaluate the real-life application of screening guidelines at an urban medical center and a practical tertiary care center, as well as its novelty; to our knowledge, there are no similar studies that evaluated the association between SES and BE surveillance adherence.

Our study has several limitations. These include the retrospective and single-center nature of the study, the inability to evaluate EGDs done at outside hospitals potentially resulting in missing data, and potential for data misclassification. Non-adherence does not necessarily translate into under surveillance, as EGDs performed and not captured by the study methodology might suggest over surveillance by some providers that is not guideline supported. Additionally, the use of zip codes to assess AGI is inferior to direct questioning of involved study patients about their annual income in a prospective fashion.

Patients with a high average AGI were more likely to be adherent with the initial surveillance endoscopy compared to patients with a low average AGI $(\mathrm{p}=0.032)$. This trend is expected, as the cost associated with endoscopy varies between $\$ 349$ and $\$ 1120[27,28]$.

\section{Conclusions}

In conclusion, higher gross income reflective of higher SES appears to be an important predictor of surveillance adherence. Although the trend approached but did not meet statistical significance on multivariate analysis, this is likely in light of the moderate sample size. Future directions include evaluating similar trends in different urban centers around the United States, combining data from multiple centers, and evaluating the impact of socioeconomic disparities in non-Caucasian populations.

\section{Additional Information \\ Disclosures}

Human subjects: Consent was obtained or waived by all participants in this study. Henry Ford Institutional Review Board issued approval NA. The Henry Ford Institutional Review Board has approved this study and waived the requirement for consent. Animal subjects: All authors have confirmed that this study did not involve animal subjects or tissue. Conflicts of interest: In compliance with the ICMJE uniform disclosure form, all authors declare the following: Payment/services info: All authors have declared that no financial support was received from any organization for the submitted work. Financial relationships: All authors have declared that they have no financial relationships at present or within the previous three years with any organizations that might have an interest in the submitted work. Other relationships: All authors have declared that there are no other relationships or activities that could appear to have influenced the submitted work.

\section{References}

1. Gatenby PAC, Caygill CPJ, Ramus JR, Charlett A, Watson A: Barrett's columnar-lined oesophagus: demographic and lifestyle associations and adenocarcinoma risk. Dig Dis Sci. 2008, 53:1175-1185. 10.1007/s10620-007-0023-y

2. Shaheen NJ, Falk GW, Iyer PG, Gerson LB: ACG clinical guideline: diagnosis and management of Barrett's esophagus. Am J Gastroenterol. 2016, 111:30-50. 10.1038/ajg.2015.322

3. van Sandick JW, Bartelsman JF, van Lanschot JJ, Tytgat GN, Obertop H: Surveillance of Barrett's oesophagus: physicians' practices and review of current guidelines. Eur J Gastroenterol Hepatol. 2000, 12:111-117.

4. Falk GW, Ours TM, Richter JE: Practice patterns for surveillance of Barrett's esophagus in the United States . Gastrointest Endosc. 2000, 52:197-203. 10.1067/mge.2000.107728

5. Ofman JJ, Shaheen NJ, Desai AA, Moody B, Bozymski EM, Weinstein WM: The quality of care in Barrett's esophagus: endoscopist and pathologist practices. Am J Gastroenterol. 2001, 96:876-881.

6. Mandal A, Playford RJ, Wicks AC: Current practice in surveillance strategy for patients with Barrett's oesophagus in the UK. Aliment Pharmacol Ther. 2003, 17:1319-1324. 10.1046/j.1365-2036.2003.01586.x

7. Amamra N, Touzet S, Colin C, Ponchon T: Current practice compared with the international guidelines: endoscopic surveillance of Barrett’s esophagus. J Eval Clin Pract. 2007, 13:789-794. 10.1111/j.13652753.2006.00754.x

8. Das D, Ishaq S, Harrison R, et al.: Management of Barrett's esophagus in the UK: overtreated and underbiopsied but improved by the introduction of a national randomized trial. Am J Gastroenterol. 2008, 103:1079-1089.

9. Abrams JA, Kapel RC, Lindberg GM, Saboorian MH, Genta RM, Neugut AI, Lightdale CJ: Adherence to biopsy guidelines for Barrett's esophagus surveillance in the community setting in the United States. Clin Gastroenterol Hepatol. 2009, 7:736-742. 10.1016/j.cgh.2008.12.027

10. Dalal KS, Coffing J, Imperiale TF: Adherence to surveillance guidelines in nondysplastic Barrett's esophagus. J Clin Gastroenterol. 2018, 52:217-222.10.1097/MCG.0000000000000777 
11. Corley DA, Kubo A, Levin TR, et al.: Race, ethnicity, sex and temporal differences in Barrett's oesophagus diagnosis: a large community-based study, 1994-2006. Gut. 2009, 58:182-188. 10.1136/gut.2008.163360

12. Khoury JE, Chisholm S, Mazen Jamal M, Palacio C, Pudhota S, Vega KJ: African Americans with Barrett's esophagus are less likely to have dysplasia at biopsy. Dig Dis Sci. 2012, 57:419-423. 10.1007/s10620-0111900-y

13. Nguyen TH, Thrift AP, Ramsey D, et al.: Risk factors for Barrett's esophagus compared between African Americans and non-hispanic whites. Am J Gastroenterol. 2014, 109:1870-1880. 10.1038/ajg.2014.351

14. Cairns CP, Viswanath K: Communication and colorectal cancer screening among the uninsured: data from the Health Information National Trends Survey (United States). Cancer Causes Control. 2006, 17:1115-1125. 10.1007/s10552-006-0046-2

15. von Wagner C, Good A, Whitaker KL, Wardle J: Psychosocial determinants of socioeconomic inequalities in cancer screening participation: a conceptual framework. Epidemiol Rev. 2011, 33:135-147. 10.1093/epirev/mxq018

16. Thein HH, Anyiwe K, Jembere N, Yu B, De P, Earle CC: Effects of socioeconomic status on esophageal adenocarcinoma stage at diagnosis, receipt of treatment, and survival: a population-based cohort study. PLoS One. 2017, 12:e0186350. 10.1371/journal.pone.0186350

17. Sampliner RE: Updated guidelines for the diagnosis, surveillance, and therapy of Barrett's esophagus . Am J Gastroenterol. 2002, 97:1888-1895.

18. Wang KK, Sampliner RE: Updated guidelines 2008 for the diagnosis, surveillance and therapy of Barrett's esophagus. Am J Gastroenterol. 2008, 103:788-797.

19. Spechler SJ, Sharma P, Souza RF, Inadomi JM, Shaheen NJ: American gastroenterological association medical position statement on the management of Barrett'S esophagus. Gastroenterology. 2011, 140:10841091. 10.1053/j.gastro.2011.01.030

20. US Income Statistics - current census data for zip codes . (2020). Accessed: March 23, 2020: https://www.incomebyzipcode.com/.

21. HAP: affordable Michigan health insurance . (2020). Accessed: March 23, 2020: https://www.hap.org/.

22. Singh M, Gupta N, Gaddam S, et al.: Practice patterns among U.S. gastroenterologists regarding endoscopic management of Barrett's esophagus. Gastrointest Endosc. 2013, 78:689-695. 10.1016/j.gie.2013.05.002

23. Dunn SJ, Neilson LJ, Hassan C, Sharma P, Guy C, Rees CJ: ESGE Survey: worldwide practice patterns amongst gastroenterologists regarding the endoscopic management of Barrett’s esophagus. Endosc Int Open. 2016, 4:E36-E41. 10.1055/s-0034-1393247

24. Akinyemiju T, Ogunsina K, Sakhuja S, Ogbhodo V, Braithwaite D: Life-course socioeconomic status and breast and cervical cancer screening: analysis of the WHO's Study on Global Ageing and Adult Health (SAGE). BMJ Open. 2016, 6:e012753. 10.1136/bmjopen-2016-012753

25. Andersen WS, Blot WJ, Lipworth L, Steinwandel M, Murff HJ, Zheng W: Association of race and socioeconomic status with colorectal cancer screening, colorectal cancer risk, and mortality in southern US adults. JAMA Netw Open. 2019, 2:e1917995. 10.1001/jamanetworkopen.2019.17995

26. Kweon SS, Kim MG, Kang MR, Shin MH, Choi JS: Difference of stage at cancer diagnosis by socioeconomic status for four target cancers of the National Cancer Screening Program in Korea: Results from the Gwangju and Jeonnam cancer registries. J Epidemiol. 2017, 27:299-304. 10.1016/j.je.2016.07.004

27. Inadomi JM, Sampliner R, Lagergren J, Lieberman D, Fendrick AM, Vakil N: Screening and surveillance for Barrett esophagus in high-risk groups: a cost-utility analysis. Ann Intern Med. 2003, 138:176-186. 10.7326/0003-4819-138-3-200302040-00009

28. Garside R, Pitt M, Somerville M, Stein K, Price A, Gilbert N: Surveillance of Barrett's oesophagus: exploring the uncertainty through systematic review, expert workshop and economic modelling. NIHR Health Technology Assessment programme: Executive Summaries. NIHR Journals Library, Southampton (UK); 2006. 